\title{
Objective measurement of cough in otherwise healthy volunteers with
}

\section{acute cough}

\author{
Kanchan Sunger*, William Powley*, Angela Kelsall`, Helen Sumner*, \\ Robert Murdoch $^{\#}$ and Jaclyn A. Smith*
}

ABSTRACT: Cough is one of the commonest reasons for medical consultation and acute cough associated with upper respiratory tract infections (URTIs) is a global problem. In otherwise healthy volunteers complaining of cough associated with symptoms of URTI, we aimed to assess objective and subjective measures of cough and their repeatability and perform power calculations for the design of future studies to test therapies.

We studied 54 otherwise healthy volunteers with acute cough ( $<3$ weeks) (median age 22 yrs (interquartile range 21-26 yrs), 64\% female, mean forced expiratory volume in $1 \mathrm{~s} 97.6 \pm 10.5 \%$ predicted). All subjects performed 24-h ambulatory cough monitoring and reported cough frequency and severity using visual analogue scales (VAS) on 2 consecutive days. Sample size calculations were performed for crossover and parallel group study designs.

Objective cough frequency was high (session 1: geometric mean 12.1 coughs $\cdot \mathrm{h}^{-1}(95 \% \mathrm{Cl}$ 9.7$15.2)$ ) and fell significantly (session 2 : 9.0 coughs $\left.\cdot h^{-1}(95 \% \mathrm{Cl} 6.9-11.6) ; p<0.001\right)$. Repeatability was higher for objective cough frequency (intra-class correlation coefficient $($ ICC) $=0.94$, $\mathrm{p}<\mathbf{0 . 0 0 1}$ ) than reported cough frequency (daytime VAS ICC $=0.784, \mathbf{p}<\mathbf{0 . 0 0 1}$ ). Crossover/parallel studies require $<\mathbf{1 5}$ and $<\mathbf{4 1}$ subjects per arm to detect a $50 \%$ reduction in cough frequency with $90 \%$ power, respectively.

Acute cough frequency is highly repeatable over any 48-h period, allowing small sample sizes to be used when investigating the effectiveness of novel anti-tussives.

KEYWORDS: Colds, cough monitoring, upper respiratory tract infections, viral infections

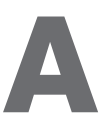
cute cough $(<3$ weeks duration) is generally caused by viral upper respiratory tract infection (URTI) [1], and cough is the commonest reason for which people seek medical attention [2-4]. On average URTIs affect adults two to four times a year [5-7] and evidence suggests that $93 \%$ of cases have an associated cough [8]. Acute cough is a global problem, costing the UK economy an estimated $£ 979$ million annually due to loss of productivity, healthcare costs and the purchase of over-the-counter (OTC) medications [9].

The mean duration of cough due to an URTI is 2.4 weeks $[8,10]$; however, such figures rely on patient reporting of cough, a potential limitation when these measures often correlate poorly with objective quantification of coughing in several other conditions [11-14]. Although acute cough is usually self-limiting and transient, like chronic cough it significantly impairs quality of life [15], and may even precipitate chronic coughing [16].

Despite the magnitude of the problem of acute cough, few studies have assessed the effectiveness of available OTC cough medicines. Most studies suggest that current therapies are no more effective than placebo, hence they cannot be recommended [17]. This, together with increasing concerns about the safety of anti-tussives in children has led the Medicines and Healthcare Products Regulatory Agency and US Food and Drug Administration to introduce restrictions in their use [18-20].

The testing of existing and novel anti-tussive therapies has, until recently, been constrained by the lack of validated tools for the assessment of coughing. However, we have found, using custom-built digital recording equipment, that it is possible to record and objectively quantify

\section{AFFILIATIONS}

${ }^{*}$ Respiratory Research Group, The University of Manchester, University Hospital of South Manchester, "Translational Research Facility, University Hospital of South Manchester, Manchester, and \#GlaxoSmithKline, Stevenage, UK.

CORRESPONDENCE

J.A. Smith

2nd Floor Education and Research Centre

University Hospital of South

Manchester

Southmoor Road

Manchester

M23 9LT

UK

E-mail: jacky.smith@

manchester.ac.uk

Received:

Nov 012011

Accepted after revision:

June 112012

First published online:

June 272012 
cough sounds over 24-h periods in ambulatory patients [21]. The study of treatments for acute cough associated with URTI is often considered particularly difficult due to its transient nature, the requirement for large parallel-designed studies and significant placebo effects [22], but the studies upon which these perceptions are based have only measured cough over periods of up to $3 \mathrm{~h}[23,24]$. The objective measurement of acute cough over longer time periods in ambulatory patients may overcome some of these difficulties. Therefore, the aims of this study were to: 1) measure objective ambulatory cough frequency in otherwise healthy adults with acute cough and symptoms suggesting a URTI; 2) establish the short-term variability of acute cough associated with URTI symptoms; 3) understand the relationships between objective and subjective measures of cough; and 4) to perform power calculations for the design of future studies of novel anti-tussive agents.

\section{MATERIALS AND METHODS \\ Subjects}

Otherwise healthy adults complaining of a cough $<3$ weeks in duration and with a history of current or preceding symptoms suggestive of a viral URTI (i.e. at least one of rhinorrhoea, sneezing, post-nasal drip, nasal congestion, sore throat, hoarse voice, fever and headache) were recruited using poster advertisements. Current smokers and ex-smokers with a smoking history of $>20$ pack-yrs were excluded, as were those with abnormal spirometry (forced expiratory volume in $1 \mathrm{~s}$ (FEV1) $<80 \%$ predicted or FEV1/forced vital capacity (FVC) $<0.7)$. Subjects taking angiotensin-converting enzyme inhibitors, codeine or other anti-tussive medicines (including OTC treatments) were also excluded. All subjects provided written informed consent and the study was approved by the local ethics committee (Tameside and Glossop 08/H1013/81; Manchester, UK).

\section{Study design}

Subjects attended the Translational Research Facility (University Hospital South Manchester, Manchester, UK) on three consecutive days. At the first attendance a medical history was collected, spirometry performed according to American Thoracic Society/European Respiratory Society standards [25] and, assuming the inclusion and exclusion criteria were met, subjects were fitted with an ambulatory cough monitor and provided with a symptom diary (session 1). Subjects attended again $24 \mathrm{~h}$ later; the cough monitor batteries and memory card were replaced and another symptom diary provided (session 2). The final visit occured a further $24 \mathrm{~h}$ later, when the cough monitor was removed and symptom diaries collected. OTC medicines were not permitted during the study period.

\section{METHODS}

\section{URTI symptoms}

At the initial visit the presence and onset of rhinorrhoea, sneezing, post-nasal drip, nasal congestion, sore throat, hoarse voice, fever and headache was documented. Subjects also reported when the cough started and the colour, frequency and volume of any sputum.

\section{Objective cough frequency}

Two 24-h ambulatory cough sound recordings were performed using a custom-built device with lapel and chest wall microphones (Vitalojak; Vitalograph Ltd, Maids Moreton, UK). The numbers of explosive cough sounds per hour [26] were counted by a single trained person (K. Sunger) using an audio editing package (Adobe Audition 3.0; Adobe, San Jose, CA, USA). We have validated this technique against cough counting from video recordings [27], and found excellent agreement between trained observers [14, 28, 29]. To facilitate manual counting, silences and low-level background noises were removed by validated, custom-written software [30]. Subjects documented the times they went to bed and got up for each recording session; these defined the periods for the day and night cough rates.

\section{Subjective cough measures}

Visual analogue scales (VAS, $100 \mathrm{~mm}$ ) were used to assess each subject's perception of the cough. For each 24-h session subjects recorded: 1$)$ cough frequency (0 $\mathrm{mm}$ : no cough, $100 \mathrm{~mm}$ : worst cough); 2) severity (0 mm: no cough, $100 \mathrm{~mm}$ : severe cough); and 3 ) difficulty falling asleep due to coughing ( $0 \mathrm{~mm}$ : not at all, $100 \mathrm{~mm}$ : couldn't sleep).

\section{Analysis}

Analyses were performed using SPSS version 15.0 (SPSS Inc, Chicago, IL, USA) and SAS version 9.2 (SAS Institute Inc., Cary, NC, USA). The primary end-point, 24-h cough frequency, was positively skewed and therefore log transformed to allow parametric analysis; for other variables non-parametric tests were applied. Repeatability between sessions was assessed using intra-class correlation coefficients (two-way, random effects). Spearman's correlation coefficients were calculated for the relationships between cough measures.

As daytime cough frequency was highly repeatable and greater than at night, the daytime cough frequency data were used to estimate variance parameters corresponding to parallel group and crossover designs. Two generalised linear mixed models were fitted to the total daytime cough count using PROC GLIMMIX (SAS Institute, Inc.). Each model had a fixed effect for recording session, used length of daytime recording as a $\log$ offset term and assumed the responses followed a negative binomial distribution (log link function). The crossover design model had an additional random subject effect term fitted on the linear predictor. The corresponding variance parameter estimates were used to obtain the standard error of a treatment effect under simple future parallel and crossover study designs. This standard error was used to compute the power of detecting a 50\% reduction in cough rate on active treatment relative to the cough rate on placebo (two-sided test, $\alpha=5 \%$ ). Since the standard error of the treatment effect (and hence power) also depends upon the placebo response, several power curves were derived to cover a range of plausible future placebo response rates. For further details see the online supplementary material.

\section{RESULTS}

\section{Subject characteristics}

54 subjects completed the study (fig. 1). Subjects had a median age of 22 yrs (interquartile range (IQR) 21.0-25.8 yrs), median body mass index $23.2 \mathrm{~kg} \cdot \mathrm{m}^{-2}$ (IQR $21.0-27.4 \mathrm{~kg} \cdot \mathrm{m}^{-2}$ ) and $64 \%$ were female. Spirometry showed a mean FEV1 of $97.6 \pm 10.5 \%$ pred and FEV1/FVC ratio of $0.85 \pm 0.08$. Two subjects were excluded with airflow obstruction, although we acknowledge that the FEV1/FVC ratio may underdiagnose airflow obstruction in a small percentage of young adults [31]. 


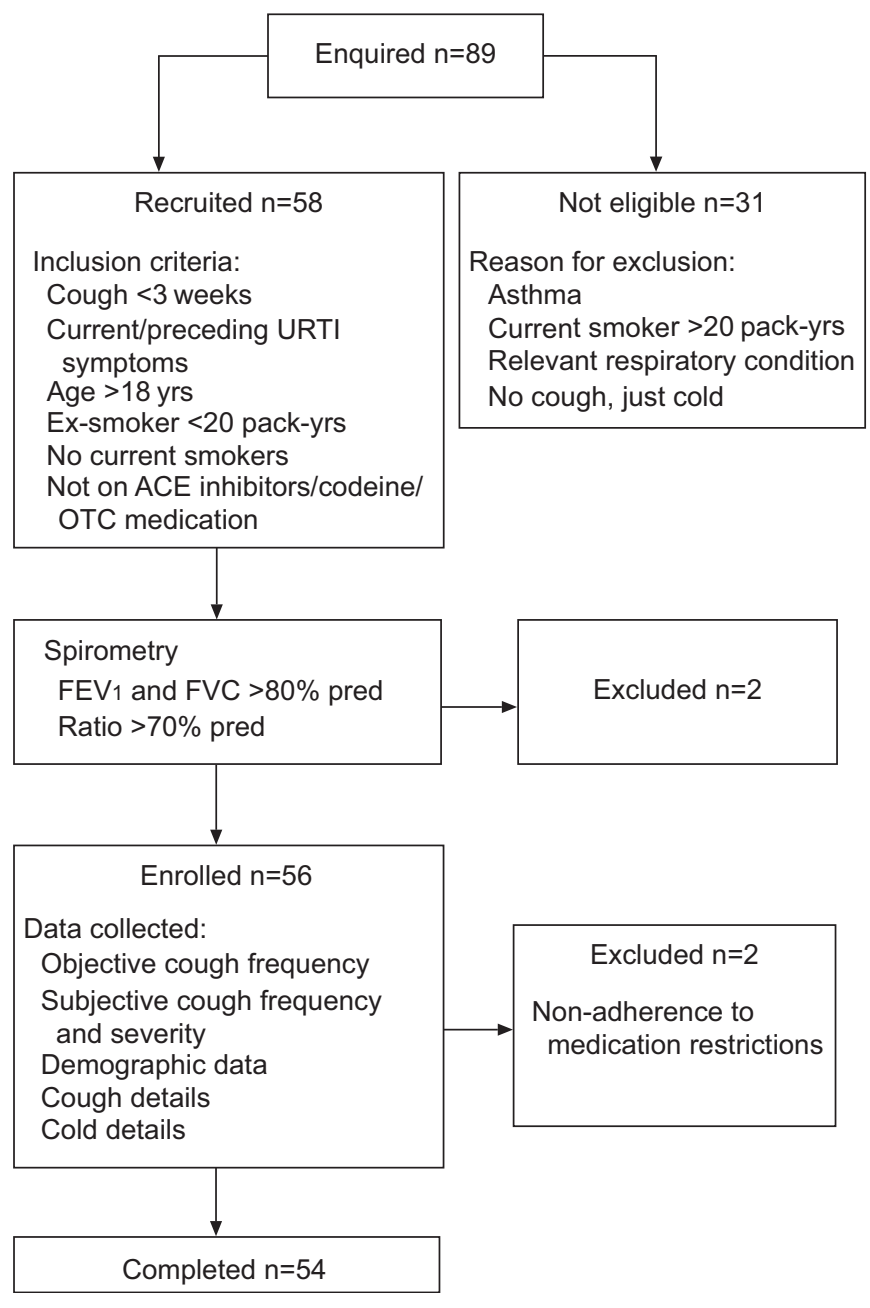

FIGURE 1. Summary of the recruitment of subjects. URTI: upper respiratory tract infection; ACE: angiotensin-converting enzyme; OTC: over-the-counter; FEV1: forced expiratory volume in $1 \mathrm{~s}$; FVC: forced vital capacity; \% pred: \% predicted.

\section{Reported URTI symptoms}

Of the reported coryzal symptoms, $79.3 \%$ had rhinorrhoea, $72.4 \%$ sneezing, $70.0 \%$ post-nasal drip, $84.5 \%$ nasal congestion, $72.4 \%$ sore throat, $44.8 \%$ fever and $55.2 \%$ headache. The median reported duration of coughing at study entry was 4 days (IQR 3.0-7.0 days) and coughing started a mean $1.4 \pm 2.2$ days after the cold started. $63.8 \%$ of subjects described a productive cough and, of these, $97.3 \%$ coughed up sputum daily and $2.7 \%$ weekly. $71.1 \%$ coughed up a teaspoon, $24.3 \%$ a tablespoon and $2.7 \%$ a cupful of sputum daily. $35.1 \%$ described the sputum as green, $27 \%$ yellow, $8.1 \%$ clear, $5.4 \%$ white and $24.3 \%$ did not know the colour.

\section{Measures of acute cough}

Objective cough frequency

A comparison of the measures of cough on both study sessions are summarised in table 1. Objective cough frequencies fell significantly from study session 1 to 2 , a median of -2.4 coughs $\cdot h^{-1}$ (IQR $-7.8--0.1$ coughs $\cdot h^{-1}$ ) and $-28.2 \%$ (IQR $-42.6--1.2 \%$ ) from baseline; see online supplementary figure E1 for a Bland-Altman plot. Considering daytime and night-time separately, only daytime cough frequency fell significantly; a median of -3.8 coughs $\cdot \mathrm{h}^{-1}$ (IQR -11.2- -0.2 coughs $\cdot \mathrm{h}^{-1}$ ) and $-28.0 \%$ (IQR $-44.3--2.7 \%$ ) from baseline. There were no significant correlations between the 24-h cough rate on either session 1 or 2 and the reported time since the cough or cold started. The difference in daytime cough rates between study sessions was also unrelated to the reported time since the cough started $(\mathrm{r}=0.05, \mathrm{p}=0.72)$ or the cold started $(\mathrm{r}=0.18, \mathrm{p}=0.18)$ (fig. 2).

Cough rates in females were not significantly different from those in males for 24-h cough frequency (geometric mean 13.7 coughs $\cdot h^{1}$ (95\% CI 10.2-18.4) versus 9.8 coughs $\cdot h(95 \%$ CI $6.8-14.1) ; \mathrm{p}=0.15)$ or day $(\mathrm{p}=0.17)$ or night $(\mathrm{p}=0.21)$. Also, there was no apparent effect of age on cough frequency $(r=0.4, p=0.79)$.

There was a marked diurnal variation in cough with substantially higher frequency during the day than overnight for both sessions $(p<0.001$ and $p<0.001$, respectively). Figure 3

TABLE 1 Summary of measures of cough on study sessions 1 and 2

\begin{tabular}{|c|c|c|c|c|}
\hline \multicolumn{5}{|c|}{ Objective cough frequency coughs $\cdot \mathrm{h}^{-1}$} \\
\hline Daytime & $19.0(9.2-31.7)$ & $13.2(5.6-26.3)$ & $-28(-44--3)$ & $<0.001$ \\
\hline Night-time & $1.7(0.3-4.2)$ & $1.3(0.2-5.1)$ & $-32(-64-77)$ & 0.51 \\
\hline \multicolumn{5}{|c|}{ Cough frequency VAS $\mathrm{mm}$} \\
\hline \multicolumn{5}{|l|}{ Cough severity VAS mm } \\
\hline Daytime & $36.0(24.3-54.0)$ & $28.5(15.5-45.0)$ & $-25(-49-8)$ & 0.18 \\
\hline Night-time & $21.0(8.0-43.8)$ & $17.5(4.0-46.3)$ & $-14^{\#}(-56-19)$ & 0.17 \\
\hline Sleep latency VAS mm & $11(2.3-22.3)$ & $10.5(1.0-30.0)$ & $-5^{\circ}(-64-80)$ & 0.77 \\
\hline
\end{tabular}



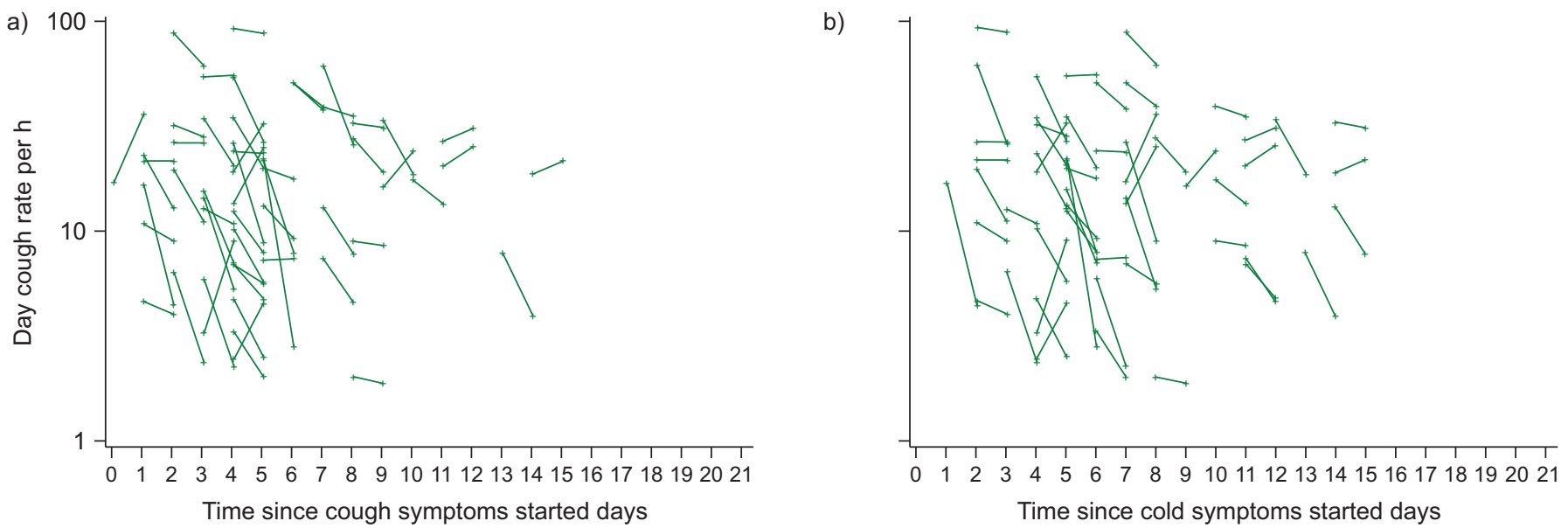

FIGURE 2. Change in daytime objective cough frequency on study session 1 and 2 in relation to reported onset of a) cough symptoms and b) coryzal symptoms. Note $\log _{10}$ scales on both $y$-axes.

shows the median cough frequency across all subjects at each time-point during the first 24 -h recording period. It is notable that cough frequency in individuals was quite variable from hour to hour, although, interestingly, the profiles of these variations tended to be similar for the two sessions (fig. 4).

\section{Subjective cough measures}

Daytime cough frequency VAS followed a similar pattern to objective cough frequency but also showed a significant change at night-time; see online supplementary figures E2 and E3 for Bland-Altman plots. In contrast, VAS measures of cough severity and sleep latency did not significantly change between sessions.

\section{Relationships between cough measures}

There were significant weak-moderate-positive correlations $(r=0.28-0.59)$ between objective cough rates and VAS measures for each study session (see online supplementary table E2 for details).

\section{Repeatability of cough measures between study sessions}

The intra-class correlation coefficients for the measures of cough are shown in table 2, suggesting that objective measures of cough are highly repeatable between the study sessions, especially daytime and 24-h cough frequency.

\section{Relationships between changes in cough measures}

Figure 5 shows the relationships between the differences in objective cough counts and cough frequency VAS from session 1 to session 2. For daytime (fig. 5a), there was only a very weak linear relationship between objective and subjective estimates of change in cough $(\mathrm{r}=0.29, \mathrm{p}=0.03)$, although the majority of subjects do appear in the left lower quadrant, i.e. both measures recorded an improvement in cough. Overnight however, most individuals cluster around the centre of the plot with little change in either measure (fig. 5b), suggesting very poor concordance between the subjective and objective changes, and no linear relationship $(\mathrm{r}=0.02, \mathrm{p}=0.90)$.

\section{Sample sizes for future study design in acute cough}

The relationships between sample sizes and power to detect a $50 \%$ reduction in daytime cough frequency (over placebo) for both crossover and parallel group studies are shown in figures $6 a$ and $b$, respectively. The influence of different placebo effects are also shown; for example, if the placebo

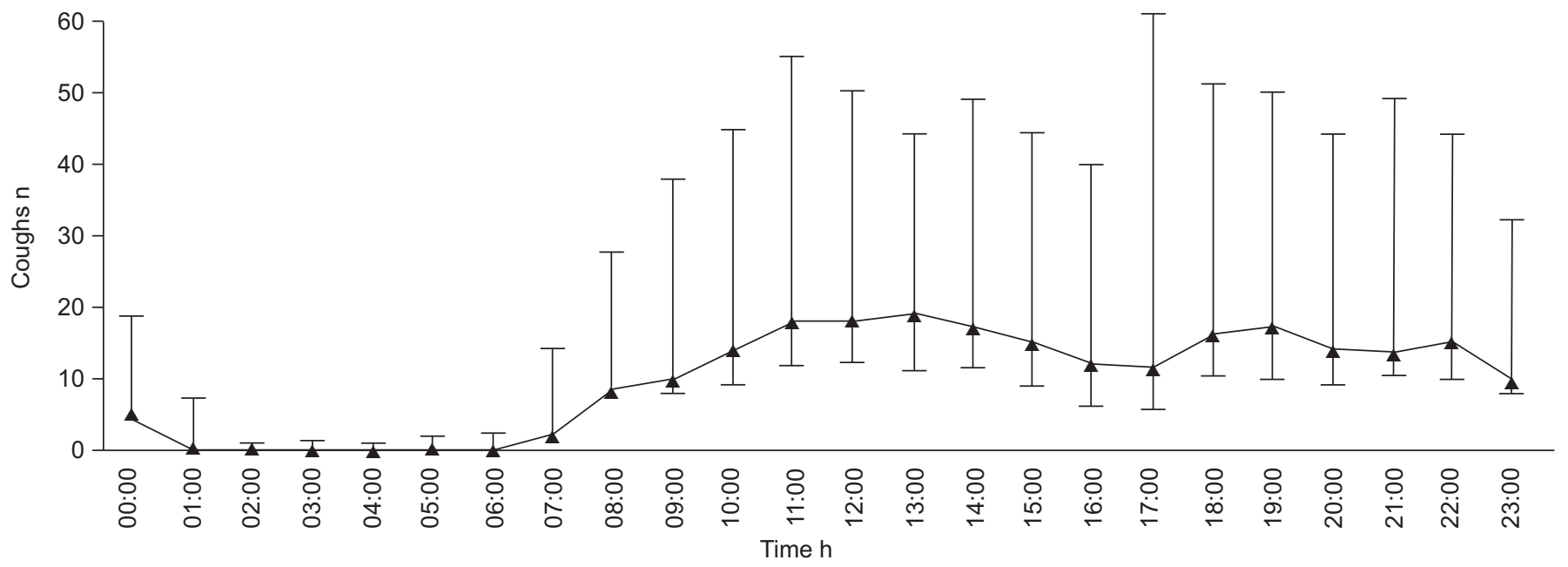

FIGURE 3. Hour-to-hour variability in cough counts for first 24-h monitoring period. Median counts are shown and error bars represent the interquartile range. 
Recording session $1 \leadsto 2$

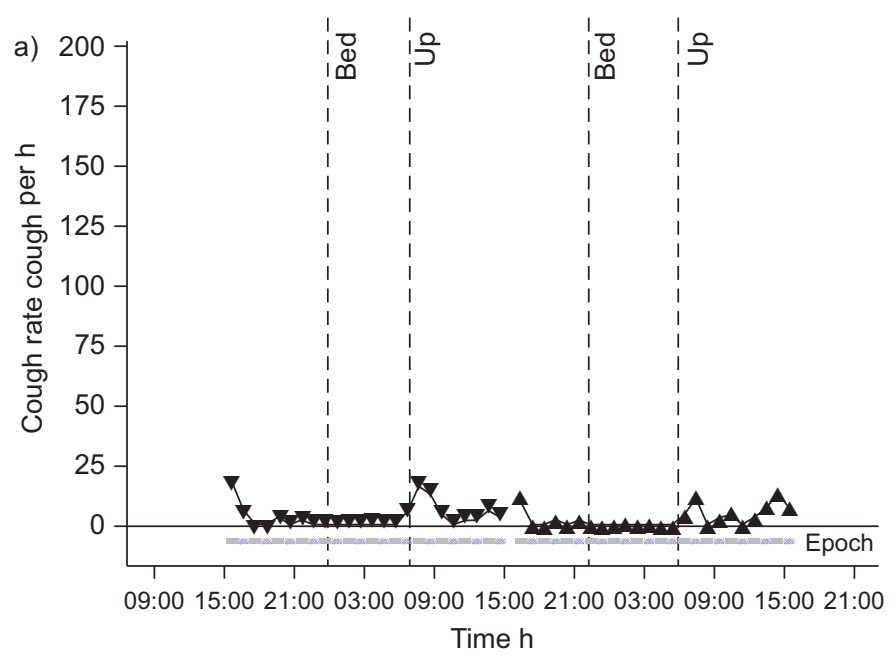

b)

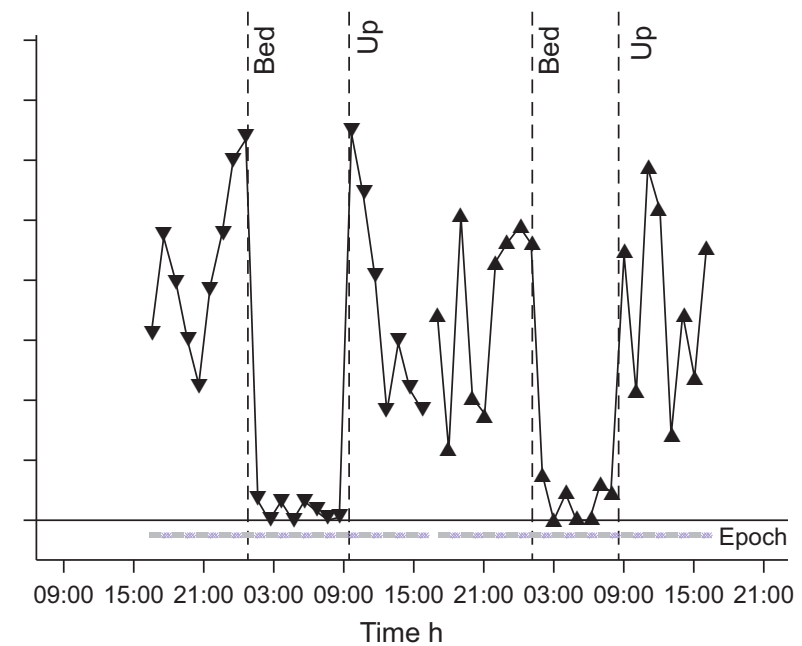

FIGURE 4. Examples of hour-to-hour variability in cough counts over both 24-h monitoring periods in two individual subjects with a) low cough rate (male aged 22 yrs, 2 days since cold onset, productive cough) and b) high cough rate (female aged 21 yrs, 2 days since cold onset, productive cough).

response rate was mean 5 coughs $\cdot h^{-1}$, the green line displays the power to detect a 2.5 coughs $\cdot h^{-1}$ reduction against the total number of subjects, whereas if the placebo cough rate was 25 coughs $\cdot h^{-1}$, the yellow line displays the power to detect a 12.5 coughs $\cdot h^{-1}$ reduction.

\section{DISCUSSION}

This is the first study to investigate 24-h objective cough frequency and its repeatability in otherwise healthy subjects complaining of an acute cough associated with URTI symptoms. Cough frequency was high and demonstrated significant variability between subjects. Although objective cough frequency fell significantly from session 1 to 2 , it was highly repeatable, especially compared with VAS ratings of cough frequency, severity and sleep latency. Furthermore, changes in objective cough frequency were poorly predicted by changes in

\begin{tabular}{|c|c|c|c|c|}
\hline \multirow{2}{*}{$\begin{array}{l}\text { TABLE } 2 \\
\text { Measure }\end{array}$} & \multicolumn{4}{|c|}{$\begin{array}{l}\text { Repeatability of cough measures from study } \\
\text { session } 1 \text { to } 2\end{array}$} \\
\hline & & ICC & $95 \% \mathrm{Cl}$ & p-value \\
\hline \multicolumn{5}{|c|}{ Objective cough frequency } \\
\hline 24-h & & 0.94 & $0.90-0.97$ & $<0.001$ \\
\hline Daytime & & 0.93 & $0.87-0.96$ & $<0.001$ \\
\hline Night-time & & 0.85 & $0.74-0.91$ & $<0.001$ \\
\hline \multicolumn{5}{|c|}{ Cough frequency VAS mm } \\
\hline Daytime & & 0.78 & $0.63-0.87$ & $<0.001$ \\
\hline Night-time & & 0.78 & $0.62-0.87$ & $<0.001$ \\
\hline \multicolumn{5}{|c|}{ Cough severity VAS mm } \\
\hline Daytime & & 0.79 & $0.64-0.88$ & $<0.001$ \\
\hline Night-time & & 0.81 & $0.68-0.89$ & $<0.001$ \\
\hline Sleep latency & VAS mm & 0.71 & $0.50-0.83$ & $<0.001$ \\
\hline
\end{tabular}

the cough frequency VAS ratings. Power calculations based on this data suggested that even considering sizeable placebo effects, anti-tussive efficacy could be observed in small numbers of subjects, especially if the characteristics of the medicine being tested allows for crossover-design studies.

It is interesting to note that the average cough frequency in acute cough was comparable to that which we have previously reported in patients presenting to a specialist clinic with chronic cough (>8 weeks duration) $[11,26]$ and in excess of rates observed in conditions such as chronic obstructive pulmonary disease [12], asthma [13] and cystic fibrosis [14]. This raises the possibility that similar mechanisms drive both acute and chronic coughing and one study has indeed suggested that one-third of chronic cough patients report their cough being initiated by an URTI [16]. Unlike in chronic cough [32], we did not observe any significant influence of age or sex on objective cough frequency in this study population, but the sample size may have been insufficient to detect these effects. This is, however, consistent with the finding that males and females with an acute cough have similar cough-specific quality-of-life scores, in contrast with chronic cough, where females have worse scores than males [15].

As might be anticipated for a viral illness, cough rates significantly dropped from session 1 to session 2, in keeping with cough resolving rapidly [8]. Whilst it is not possible to determine the mechanisms underlying the fall in cough frequency from this study, it is important to appreciate that objective cough frequency was still highly repeatable. This may seem contradictory, but the drop in cough rate was consistent across the range of cough frequencies at session 1, and the rank order of the patients (in terms of cough rates) remained very similar for session 2; therefore, although the absolute cough rates fell, the variability in cough frequency within subjects (between sessions 1 and 2) was much less than the variability between subjects (within each session), resulting in high intraclass correlation coefficients and repeatability. This finding has 

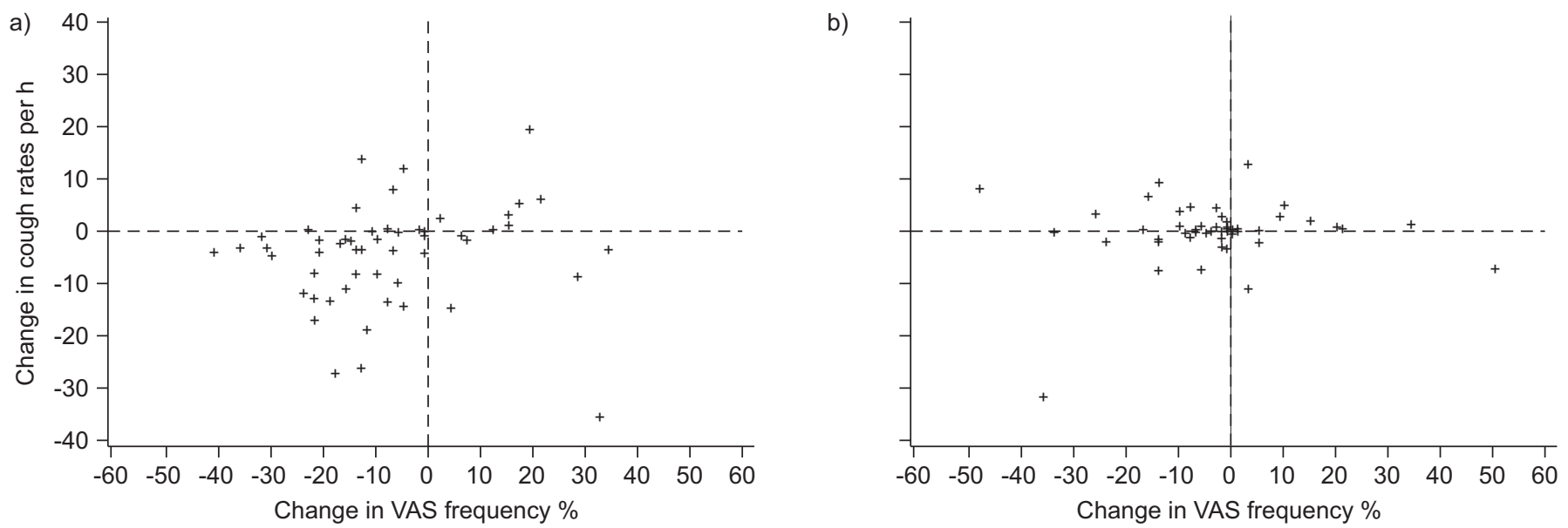

FIGURE 5. Relationship between differences in objective cough frequency and cough visual analogue scale (VAS) from study session 1 to 2 for a) daytime and b) night-time.

important implications for future trial design, suggesting that crossover studies utilising objective cough frequency measures are feasible over a 48 -h period. Such a study design would only be appropriate for short-acting agents with rapid onset/offset and equal efficacy in patients, irrespective of their baseline cough rates; such characteristics are, however, likely to be desirable in a therapy for acute cough. It is also noteworthy that the reported onset date of the cough or cold symptoms did not predict the objective cough frequency, suggesting that the trajectory of objective cough counts in viral illness is highly variable. Within the 3 -week time window for acute cough, it is therefore unnecessary to target patients with any particular reported symptom duration for recruitment.

The importance of the placebo effect in the treatment of acute cough has been frequently highlighted, and may be responsible for up to $85 \%$ of the efficacy of some cough medicines [24]. These large effects have generally been found under laboratory conditions, monitoring cough over just 15-min periods. Such

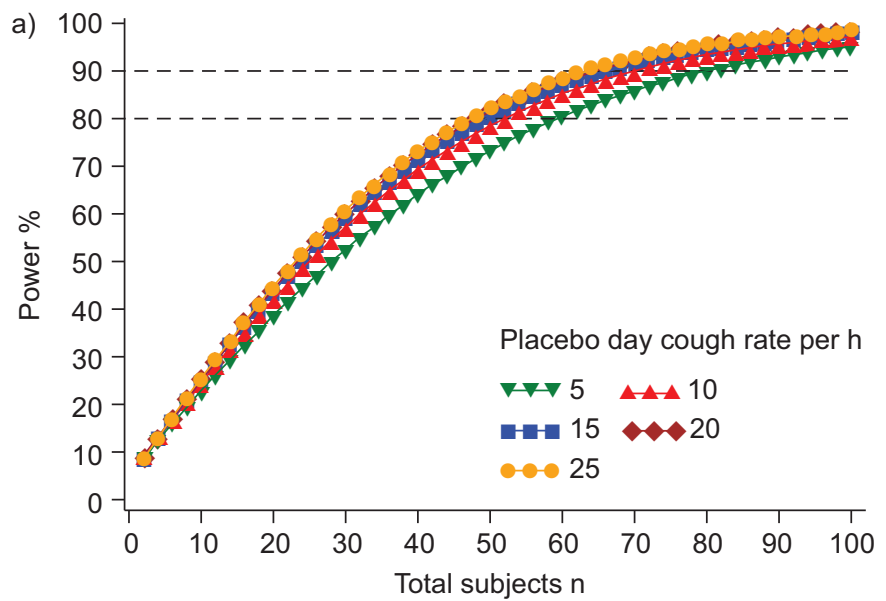

conditions might be expected to exaggerate placebo effects compared with monitoring over $24 \mathrm{~h}$ in a subject's own environment. Nonetheless, when performing power calculations from this data we included a wide range of placebo means (representing potential outcomes of small and large placebo effects) and still found sample sizes to be relatively small.

For our power calculations, we estimated that an improvement of $50 \%$ in objective cough frequency may be required for patients to appreciate an improvement in their cough. Studies with effective medicines are needed to clarify this issue; however, in chronic cough patients undergoing reflux monitoring with a naso-gastric catheter a fall of one-third in objective cough frequency was accompanied by a significant improvement in cough VAS, suggesting that this change was perceived as an improvement [33]. In the absence of data specific to acute cough, we estimated that a slightly larger change (i.e. 50\% fall) might be necessary for an improvement in coughing to be appreciated; however, this figure may differ significantly across conditions.

b)

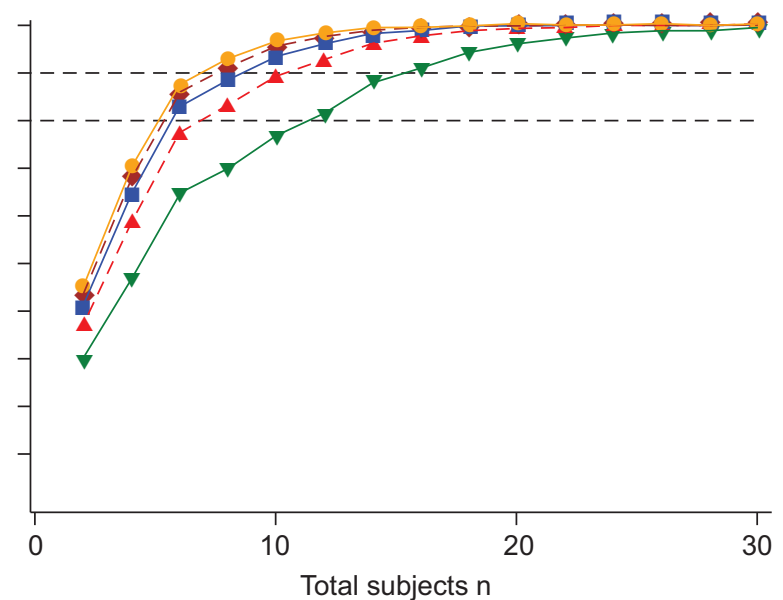

FIGURE 6. Sample size calculations in acute cough for both a) parallel- and b) crossover-designed studies. Graphs display the balance between power and the total number of subjects required to detect a $50 \%$ reduction in cough frequency over placebo, calculated for a range of plausible cough rates on placebo therapy. Note that for parallel designs (a), each treatment arm would require half the number of subjects shown. 
This study has some limitations. First, our study population was mainly young adults, and therefore it remains to be determined whether cough frequencies may be different in older adults with URTI symptoms. Epidemiological data would suggest that the incidence of URTI tends to be inversely correlated with age and that the sample we studied (adults in their early 20s) are representative of the age group most frequently affected by URTI episodes (apart from children) [34, 35]. Secondly, our sample contained more females than males. It is known that more females present to general practice with coughs secondary to URTI [34], which may also explain the excess of females presenting for inclusion in this study. Indeed other studies of treatments for acute cough have often recruited a similar proportion of females [36-38]. Finally it remains to be seen whether limiting recruitment to patients earlier in the natural history of colds has any significant impact on the variability and repeatability of cough frequency.

In summary, this study shows that acute cough rates are comparable to those found in chronic cough and highly repeatable over any 48 - $\mathrm{h}$ period. These findings have significant implications for the testing of novel anti-tussive agents, demonstrating that objective cough frequency monitoring is a more powerful tool than subjective measures of acute cough, reducing the number of subjects required and making crossover designs possible.

\section{SUPPORT STATEMENT}

The study was funded by GlaxoSmithKline and the Medical Research Council. J. Smith is funded by a Clinician Scientist Fellowship from the Medical Research Council.

\section{STATEMENT OF INTEREST}

Statements of interest for W.M. Powley, R. Murdoch and J.A. Smith, and for the study itself can be found at www.erj.ersjournals.com/site/ misc/statements.xhtml

\section{ACKNOWLEDGEMENTS}

We wish to acknowledge the contribution of all the volunteers who took part and also the funding provided by GlaxoSmithKline.

\section{REFERENCES}

1 Worrall G. Acute cough in adults. Can Fam Physician 2011; 57: $48-51$.

2 Schappert SM. National Ambulatory Medical Care Survey: 1991 summary. Vital Health Stat 13 1994; 1-110.

3 Burt CW, Schappert SM. Ambulatory care visits to physician offices, hospital outpatient departments, and emergency departments: United States, 1999-2000. Vital Health Stat 13 2004; 1-70.

4 National Ambulatory Medical Care Survey: 2009 summary tables. Hyattsville, Ambulatory and Hospital Care Statistics Branch National Center for Health Statistics, 2009.

5 Boujaoude ZC, Pratter MR Clinical approach to acute cough. Lung 2010; 188 Suppl. 1, S41-S46.

6 Heikkinen T, Jarvinen A. The common cold. Lancet 2003; 361: 51-59.

7 Monto AS. Studies of the community and family: acute respiratory illness and infection. Epidemiol Rev 1994; 16: 351-373.

8 Jones BF, Stewart MA. Duration of cough in acute upper respiratory tract infections. Aust Fam Physician 2002; 31: 971-973.

9 Morice AH, McGarvey L, Pavord I. Recommendations for the management of cough in adults. Thorax 2006; 61: Suppl. 1, i1-i24.
10 Curley FJ, Irwin RS, Pratter MR, et al. Cough and the common cold. Am Rev Respir Dis 1988; 138: 305-311.

11 Decalmer SC, Webster D, Kelsall AA, et al. Chronic cough: how do cough reflex sensitivity and subjective assessments correlate with objective cough counts during ambulatory monitoring? Thorax 2007; 62: 329-334.

12 Smith J, Owen E, Earis J, et al. Cough in COPD: correlation of objective monitoring with cough challenge and subjective assessments. Chest 2006; 130: 379-385.

13 Marsden PA, Smith JA, Kelsall AA, et al. A comparison of objective and subjective measures of cough in asthma. J Allergy Clin Immunol 2008; 122: 903-907.

14 Smith JA, Owen EC, Jones AM, et al. Objective measurement of cough during pulmonary exacerbations in adults with cystic fibrosis. Thorax 2006; 61: 425-429.

15 French CT, Fletcher KE, Irwin RS. A comparison of gender differences in health-related quality of life in acute and chronic coughers. Chest 2005; 127: 1991-1998.

16 Haque RA, Usmani OS, Barnes PJ. Chronic idiopathic cough: a discrete clinical entity? Chest 2005; 127: 1710-1713.

17 Schroeder K, Fahey T. Systematic review of randomised controlled trials of over the counter cough medicines for acute cough in adults. BMJ 2002; 324: 329-331.

18 Smyth R. Updated advice - over-the-counter cough and cold medicines for children. Drug Safety Update 2008; 9: 233-242.

19 MHRA. Codeine-containing liquid over-the-counter medicines: should not be used for cough under 18 years. Drug Safety Update. Volume 4, issue 3, October 2010. www.mhra.gov.uk/ Safetyinformation/DrugSafetyUpdate/CON096805.

20 Kuehn BM. Citing serious risks, FDA recommends no cold and cough medicines for infants. JAMA 2008; 299: 887-888.

21 Smith J. Ambulatory methods for recording cough. Pulm Pharmacol Ther 2007; 20: 313-318.

22 Pavord ID, Chung KF. Management of chronic cough. Lancet 2008; 371: 1375-1384.

23 Pavesi L, Subburaj S, Porter-Shaw K. Application and validation of a computerized cough acquisition system for objective monitoring of acute cough: a meta-analysis. Chest 2001; 120: 1121-1128.

24 Eccles R. Importance of placebo effect in cough clinical trials. Lung 2010; 188: Suppl. 1, S53-S61.

25 Miller MR, Hankinson J, Brusasco V, et al. Standardisation of spirometry. Eur Respir J 2005; 26: 319-338.

26 Kelsall A, Decalmer S, Webster D, et al. How to quantify coughing: correlations with quality of life in chronic cough. Eur Respir J 2008; 32: $175-179$

27 Smith JA, Earis JE, Woodcock AA. Establishing a gold standard for manual cough counting: video versus digital audio recordings. Cough 2006; 2: 6.

28 Smith JA, Decalmer S, Kelsall A, et al. Acoustic cough-reflux associations in chronic cough: potential triggers and mechanisms. Gastroenterology 2010; 139: 754-762.

29 Key AL, Holt K, Hamilton A, et al. Objective cough frequency in idiopathic pulmonary fibrosis. Cough 2010; 6: 4.

30 Sumner H, Kelsall A, Woodcock A, et al. A semi-automatic method to reduce the time taken for manual cough counting. Am J Resp Crit Care Med 2010; 181: A5555.

31 Cerveri I, Corsico AG, Accordini S, et al. Underestimation of airflow obstruction among young adults using FEV1/FVC $<70 \%$ as a fixed cut-off: a longitudinal evaluation of clinical and functional outcomes. Thorax 2008; 63: 1040-1045.

32 Kelsall A, Decalmer S, McGuinness K, et al. Sex differences and predictors of objective cough frequency in chronic cough. Thorax 2009; 64: 393-398.

33 Kelsall A, Houghton LA, Jones $\mathrm{H}$, et al. A novel approach to studying the relationship between subjective and objective measures of cough. Chest 2011; 139: 569-575. 
34 Fleming DM, Elliot AJ, Nguyen-Van-Tam JS, et al. A Winter's Tale: Coming to Terms with Winter Respiratory Illnesses. London, Health Protection Agency, 2005.

35 Gonzales R, Malone DC, Maselli JH, et al. Excessive antibiotic use for acute respiratory infections in the United States. Clin Infect Dis 2001; 33: 757-762.

36 Freestone C, Eccles R. Assessment of the antitussive efficacy of codeine in cough associated with common cold. J Pharm Pharmacol 1997; 49: 1045-1049.
37 Lee PCL, Jawad MS, Eccles R. Antitussive efficacy of dextromethorphan in cough associated with acute upper respiratory tract infection. J Pharm Pharmacol 2000; 52: 1137-1142.

38 Prasad AS, Beck FW, Bao B, et al. Duration and severity of symptoms and levels of plasma interleukin-1 receptor antagonist, soluble tumor necrosis factor receptor, and adhesion molecules in patients with common cold treated with zinc acetate. J Infect Dis 2008; 197: 795-802. 